\title{
The Research of Forest Fire Smoke Moving Target Detection Methods Based on Video Streaming
}

\author{
Zhijian Yin ${ }^{1, a}$, Kun Xiang ${ }^{1, b}$, Zhaopan Wu ${ }^{1, c}$, Qiang Luo ${ }^{1, d}$ and Wang Fan ${ }^{1, e}$ \\ ${ }^{1}$ School of Communication and Electronic, Jiangxi Science \& Technology Normal University, \\ Nanchang, 330013, China \\ aemail: 86412656@qq.com, bemail: 1244689313@qq.com, cemail: 654591305@qq.com, \\ demail: 13576918019@163.com, eemail:1245905084@qq.com
}

Keyword: Moving target detection; Inter-frame Difference Method; Background Estimation Method; Color Judgment

\begin{abstract}
Moving target detection is the premise to classify and recognize the video images correctly. The smoke is a marked characteristic in the early forest fire. Through the analysis of the characteristics of forest fire smoke image, this paper studied several common moving target detection methods, analyzed their realization processes, compared their advantages and disadvantages, and sought the best method of video forest fire smoke moving target detection. Experiments showed that the improved background estimation method combined with color judgment criteria not only has better ability of smoke capture, but also has stronger anti-interference ability, which greatly eases the pressure on the subsequent image recognition.
\end{abstract}

\section{Introduction}

Moving target automatic detection is one technology to detect, extract, recognize and track moving target[1].In the video image sequences of forest fire, the effect of smoke moving target detection directly affects the late feature extraction and recognition. The great environmental adaptation ability is a basic requirement for the moving target detection algorithm with good effect, such as processing shadow and occlusion, adapting to changes in light, etc. And good real-time performance is also an aspect of good detection effects. Hence, the complexity, reliability and environmental adaptation ability of algorithm should be taken in account in the specific application.

As one hot and difficulty in the current research, the research of moving target detection becomes more difficult because of the complex and volatile forest environment. The forest video monitoring includes static monitoring and dynamic monitoring, and the later was used in the most cases. According to the smoke characteristics of color and diffusivity, this paper studied several moving target detection methods under the static background, and discussed these problems of the background update, the light change and the distractor disturbance.

\section{The Common Detection Methods}

At present, there are many kinds of moving target detection methods, but different detection methods should be adopted in different practical applications. In this paper, the forest fire smoke moving target detection methods based on video streaming are studied, whose purpose is to seek the best smoke moving target detection method by analyzing their realization processes and comparing their advantages and disadvantages.

\section{Inter-frame Difference Method}

Inter-frame difference method is a classic algorithm which is rapid, relatively accurate and used widely. In this paper, the suspected smoke area detection in the forest fire image is realized by inter-frame difference method for continuous frames. And the specific process is to take difference operation for different frames firstly, set the gray threshold, transform the difference image into binary image and extract the suspected moving area by moving areas. The flow chart of inter-frame 
difference method is shown in the Fig. 1. The specific steps are as follows:

(1) To extract the frame sequences in the video by sampling and the sampling rate is $1 \mathrm{FPS}$;

(2) To preprocess the sampled images and take difference operation for two adjacent frames;

(3) To transform the difference image into binary image by setting the threshold $\mathrm{T}=90$, and circle the moving area by rectangular box.

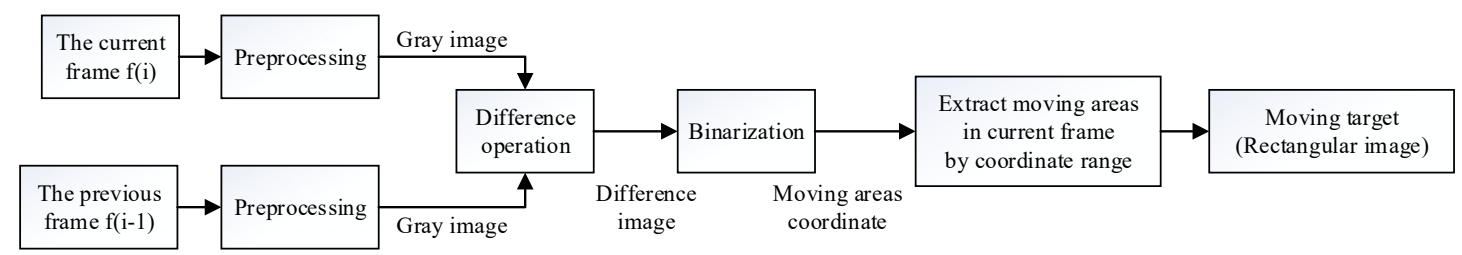

Fig. 1 The flow chart of inter-frame difference method

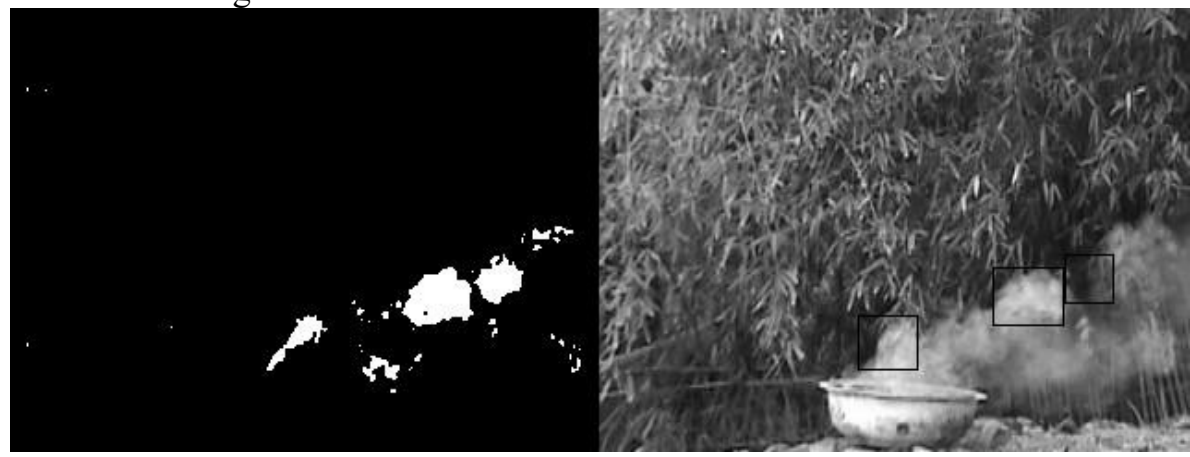

Fig. 2 The extraction effect of image moving areas

The Fig. 2 shows the extraction effect of image moving areas. Experiments show that inter-frame difference method cannot well filter out the non-smoke moving objects, such as pedestrians and birds. And when there are slight changes with a wide range in the video images, this method cannot separate the moving body from the image correctly.

\section{Background Estimation Method}

Background estimation method not only has the characteristic of rapid calculation which is same to inter-frame difference method, but also improves filtering non-moving body's slight moving. The algorithm introduces the background mechanism, and it takes difference operation for some frame with the background frame, rather than two adjacent frames, which makes up the deficiency of inter-frame difference method, and improves the effect of target extraction. The flow chart of background estimation method is shown in Fig. 3. The first frame in the initial sequence is regarded as the initial background, and the background updating formula is as follows:

$$
B_{n+1}(x, y)=\left\{\begin{array}{cc}
B_{n}(x, y) & (x, y) \text { stationary } \\
a B_{n}(x, y)+(1-a) I_{n+1}(x, y) & (x, y) \text { moving }
\end{array}\right.
$$

In the formula, $B_{n+1}(x, y)$ means the gray value of the coordinate $(x, y)$ in $\mathrm{n}+1$ frame background image, $I_{n+1}(x, y)$ means the gray value of the coordinate $(x, y)$ in $\mathrm{n}+1$ frame of sampling video sequence, and $a$ means weight coefficient. Through experimental comparison, this paper sets $\mathrm{a}=0.6$, which ensures the capture ability of moving body not to be affected, and can filter out the slight disturbance in the image successfully.

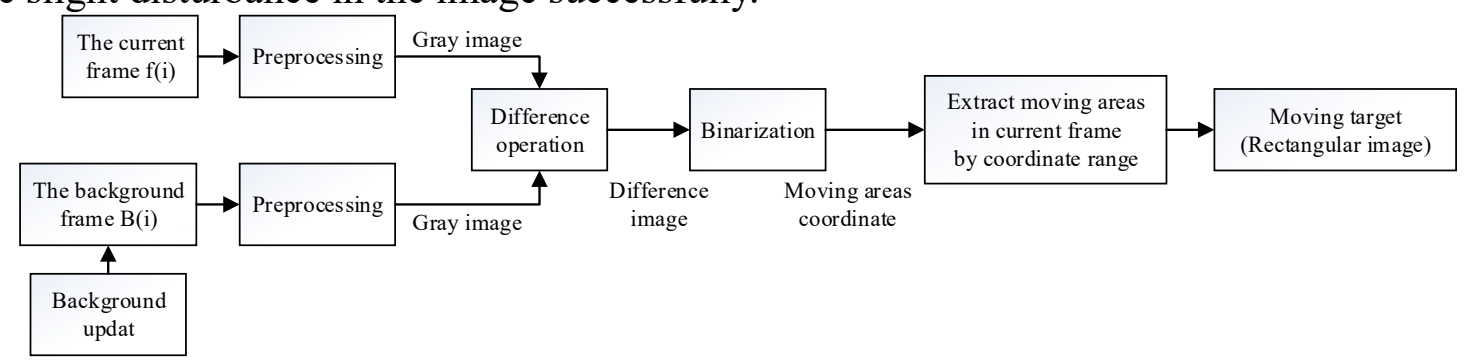

Fig. 3 The flow chart of background estimation method 


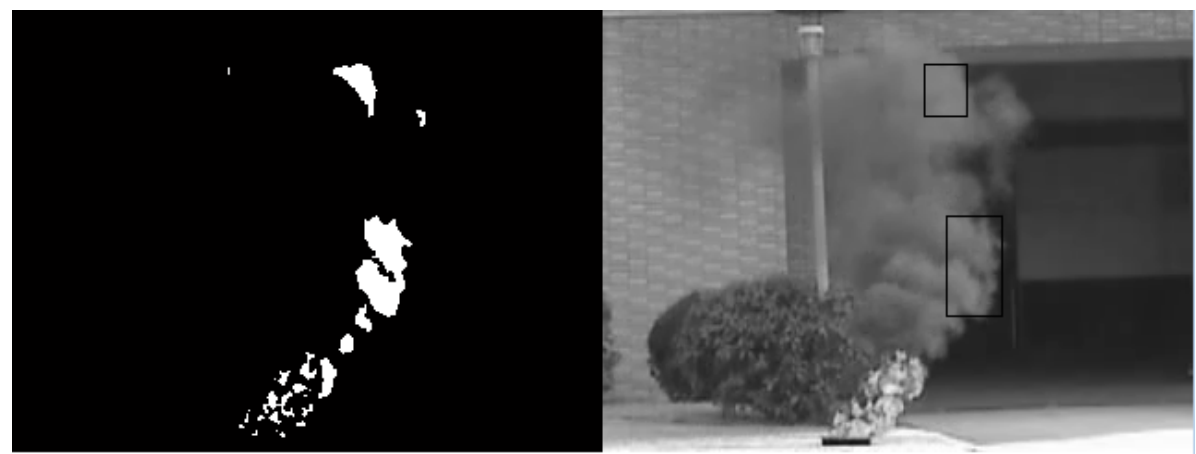

Fig. 4 Smoke target extraction by background estimation method

Smoke target extraction by background estimation method is shown in Fig. 4. The experimental results show that background estimation method solves well the deficiency of inter-frame difference method. This method with relatively large $a$ value filters out the slight disturbance, but it results that slow-moving smoke itself will also be wrong to exclude, which can cause omission. However, inter-frame difference method and background estimation method with a small $a$ value both cause that other distractors are regarded as smoke wrongly.

\section{The Improved Background Estimation Method Combined with Color Judgment Criteria}

To make up the defects of the above two methods, background estimation method is improved according to smoke's diffusivity. The improved method changes the background updating formula by introducing initial background, which can achieve better smoke separation and distractors filtration.

With the increase of time interval, the diffusivity makes the difference between different frames bigger, which is different from the slight disturbance of distractors. As a long time interval background reference, the initial background is used to update the background image, which won't regard the smoke that changes slowly as the background in the detection process. This is the principle of the improved background updating method. The improved updating formula is as follows:

$$
B_{n+1}(x, y)=\left\{\begin{array}{cc}
B_{n}(x, y) & (x, y) \text { stationary } \\
a B_{n}(x, y)+b I_{n+1}(x, y)+(1-a-b) B_{1}(x, y) & (x, y) \text { moving }
\end{array}\right.
$$

In the formula, $B_{1}(x, y)$ means the gray value of the coordinate $(x, y)$ in the initial background of first frame, $a$ and $b$ are weight coefficient, and $a+b<1$. Through experiment contrast, this paper sets $a=0.4$ and $b=0.3$ respectively in the new algorithm, which not only has better capture ability for slow-moving smoke, but also can filter out the slight disturbance of distractors successfully. Smoke target extraction by the improved background estimation method is shown in Fig. 5.

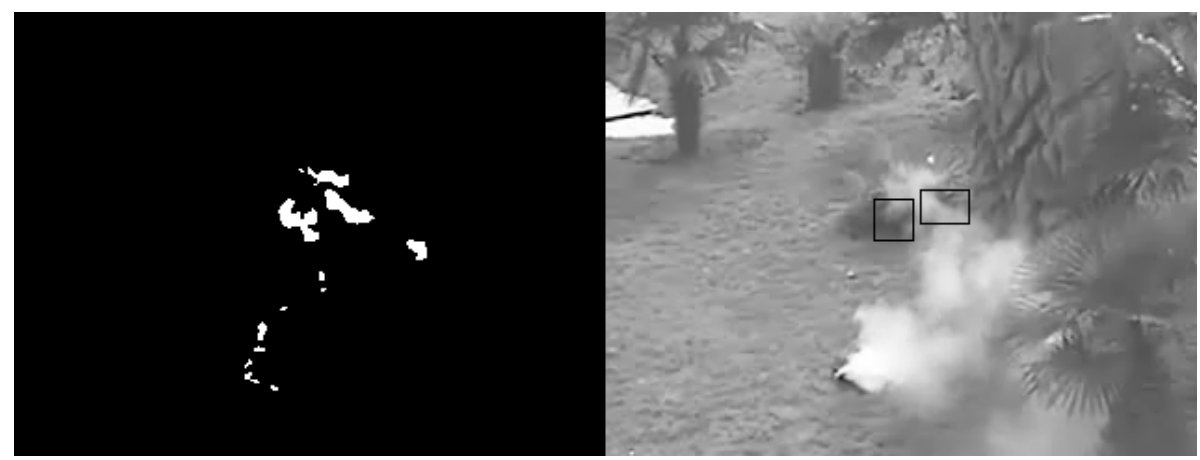

Fig. 5 Smoke target extraction by the improved background estimation method

Color is the most intuitionistic feature in many features of forest fire smoke, and it is mainly gray or similar to gray. According to this feature, the human can recognize easily that some certain colors are not the suspected smoke obviously. So the color judgment can be used as a supplementary 
means for filtering out non-smoke moving body.

From Fig. 6, it is easy to know that the new algorithm combines the improved background estimation method with color judgment criteria, which filters out some non-smoke disturbances after the moving areas are detected and reduces the workload in the static feature extraction and pattern recognition of subsequent stages. But the fog is extremely similar to smoke on color. It is difficult to rule out fog by color judgment criteria.

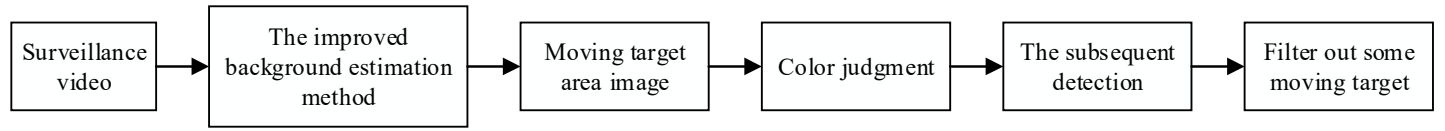

Fig. 6 The flow chart of the improved background estimation method combined with color judgment criteria

\section{The Experimental Contrast}

This paper compares these four methods from four aspects of the ability of smoke capture, the ability of filtering out the disturbances, the algorithm performance and the ability of filtering out color moving areas respectively. And the results are shown in Table 1.

Table 1 The result of experimental comparison about four methods

\begin{tabular}{|c|c|c|c|c|}
\hline Method & $\begin{array}{c}\text { Inter-frame } \\
\text { difference method }\end{array}$ & $\begin{array}{c}\text { Background } \\
\text { estimation method }\end{array}$ & $\begin{array}{c}\text { The improved } \\
\text { background } \\
\text { estimation method }\end{array}$ & $\begin{array}{c}\text { The improved } \\
\text { background estimation } \\
\text { method with color } \\
\text { judgment }\end{array}$ \\
\hline Smoke capture & Good & General & Good & Good \\
\hline $\begin{array}{c}\text { Filter out } \\
\text { interference }\end{array}$ & General & Good & Good & Good \\
\hline $\begin{array}{c}\text { Algorithm } \\
\text { performance } \\
\text { (1 FPS) }\end{array}$ & $9.14 \mathrm{~s}$ & $12.15 \mathrm{~s}$ & $12.29 \mathrm{~s}$ & $12.61 \mathrm{~s}$ \\
\hline $\begin{array}{c}\text { Filter out color } \\
\text { moving areas }\end{array}$ & No & No & No & Yes \\
\hline
\end{tabular}

It can be known from Table 1 that inter-frame difference method is similar to the improved background estimation method on smoke capture and both are better than the initial. And background estimation method and the improved are better greatly than inter-frame difference method on filtering out interference. What's more, on algorithm performance, inter-frame difference method whose model is most simple runs fastest and other three algorithms are similar. It is worth noticing that the improved background estimation method combined with color judgment criteria is best on the whole. It has better smoke capture ability, can filter out well slow-moving disturbances, and rule out the disturbances of great-shaking trees and color mobile cars successfully, which not only improves the anti-interference ability of system and reduces the work of extraction and recognition in the late.

\section{Conclusion}

In this paper, four moving target detection algorithms were studied about forest fire smoke image. There are inter-frame difference method, background estimation method, the improve background estimation method and the improved background estimation method combined with color judgment criteria respectively. And their detection effects can be known by four specific comparisons. The improved background estimation combined with color judgment criteria is best on the whole, which reduces the pressure of image recognition in the late and improves the anti-interference ability of the system. Although this method rule out the interference of some cloud and fog well, it is very difficult to realize in cloudy and foggy areas. So this moving target detection algorithm should be improved further. 


\section{Reference}

[1] Yanqi Liu , Fuyu Zhan. Practical Works Instruction of Image and Video Processing Based on MTALAB[M], Beijing, 2015: 208-218.

[2] Rule Huang. Research on Forest Fire Smoke Recognition Based on Video Images[D], Beijing, 2011: 18-56.

[3] Qiuqi Ruan. Digital Image Processing (3rd Edition)[M], Beijing, 2013: 25-89.

[4] Fan Yang, Zhitao Wang, Hua Zhang. Proficient in Image Processing Classic Algorithms (MATLAB version)[M], Beijing, 2014: 11-63.

[5] Chendan Shi. The Research of Forest Fire Monitoring Methods and System Implementation Based on Video Image[D], Nanjing, 2013: 8-23. 